\title{
Eficacia del uso de bioconectores para los catéteres centrales de hemodiálisis
}

\author{
"Premio Covidien Quinton al mejor trabajo de investigación sobre catéteres permanentes \\ 2010"
}

Ma Amor Martínez Aranda - Patricia Arribas Cobo - Ana Ruiz Álvarez - Jaime Rodríguez Estaire - Irene Callejo Cano - Pilar Díaz de Argote - Alfredo Sáenz Santolaya - Raquel Cerrajero Calero - Beatriz Liébana Pamos - $\mathbf{M}^{\mathrm{a}}$ Ángeles Guimerá Ferrer-Sama - Manuela Larico Ccuno - $\mathbf{M}^{\mathrm{a}}$ Trinidad Quesada Armenteros - Elena Herrera Martín - Gema Vinagre Rea - Sonia García Estévez

Diplomados Universitarios en Enfermería

Unidad de diálisis. Hospital Universitario Infanta Leonor. Madrid

\section{Resumen}

Los catéteres tunelizados (CT) constituyen el acceso vascular de hemodiálisis (HD) en un porcentaje elevadísimo de pacientes.

La utilización de CT se asocia a dos problemas fundamentales: disfunción e infecciones.

Según las guías de la Sociedad Española de Nefrología (SEN), en los pacientes en HD se aconseja el empleo de tapones de un solo uso con rosca de seguridad (luer-lock). En el mercado existen también bioconectores, que son dispositivos mecánica y microbiológicamente cerrados. La ventaja que ofrecen es la menor manipulación de la luz del catéter al cambiarse una vez por semana.

Se realizó un estudio en nuestro centro analizando en una muestra de 14 pacientes portadores de CT, dos periodos consecutivos, un primer periodo siguiendo el protocolo de conexión convencional y el segundo utilizando los bioconectores TEGO. Se analizaron

Correspondencia:

Unidad de Diálisis

Hospital Universitario Infanta Leonor

Avd. Gran Vía del Este 80, 28031 Madrid

E-mail:mamor.martinez@salud.madrid.org

E-mail:parribasc@salud.madrid.org presiones arterial y venosa, flujos sanguíneos, $\mathrm{Kt}$, $\mathrm{Kt} / \mathrm{v}$ y tasa de bacteriemia.

No se obtuvieron diferencias significativas en estos parámetros con el uso de dispositivos TEGO frente al uso convencional.

Podemos concluir que el uso de tapones TEG0 no afecta a los parámetros dialíticos. No obstante, debemos considerar el manejo de cualquier tipo de dispositivo como secundario en la prevención de bacteriemias, ya que se ha demostrado que son las medidas universales de asepsia las que logran una tasa de bacteriemias muy baja.

PALABRAS CLAVE:

- CATÉTER

- BIOCONECTOR

- BACTERIEMIA

- HEMODIÁLISIS

Efficacy of the use of bioconnectors for central catheters in haemodialysis

\section{Abstract}

Tunnelled catheters (TC) are the vascular access used in haemodialysis (HD) for a very high percentage of patients. 
The use of TC is associated to two fundamental problems: dysfunction and infections.

According to the guidelines established by the Spanish Nephrology Society (SEN), the use of luer-lock catheters is recommended in HD patients. On the market there are also bioconnectors, which are mechanically and microbiologically sealed devices. The advantage they offer is better handling of the catheter lumen when changed once a week.

A study was carried out at our centre analysing two consecutive periods in a sample of 14 patients with CT. In the first period the conventional connection protocol was followed and in the second TEGO bioconnectors were used. Arterial and venous blood pressure was measured, blood flows, $\mathrm{Kt}, \mathrm{Kt} / \mathrm{v}$ and bacteraemia rate.

No significant differences were obtained in these parameters with the use of TEGO devices compared to conventional use.

We may conclude that the use of TEGO locks does not affect the dialytic parameters. However, we should consider the handling of any type of device as secondary in the prevention of bacteraemia, as it has been proved that universal aseptic measures are what achieve a very low bacteraemia rate.

\section{KEY WORDS:}

- CATHETER

- BIOCONNECTOR

- BACTERAEMIA

HAEMODIALYSIS

\section{Introducción}

Los catéteres tunelizados (CT), pese a las recomendaciones de todas las sociedades nefrológicas ${ }^{1}$, constituyen un porcentaje elevadísimo de los accesos vasculares en hemodiálisis (HD). Así, el estudio reciente de la Sociedad Madrileña de Nefrología muestra que el 45\% de los pacientes inician HD con un catéter y que el CT es el acceso utilizado en el $29,5 \%$ de los pacientes prevalentes en $\mathrm{HD}$, sin que en los últimos años haya disminuido su uso².

La utilización de CT se asocia a dos problemas fundamentales: disfunción e infecciones. Aunque la infección es menos frecuente, la bacteriemia relacionada con el catéter (BRC) causa una elevada morbimortalidad, mediada por la aparición de infecciones metastásicas (3,2-50\% según series) y de sepsis severa, lo que depende en gran medida del germen implicado, ya que el riesgo es mayor si están causadas por S. Aureus. No hay que olvidar que las infecciones son la segunda causa de mortalidad en $\mathrm{HD}^{3}$.

La infección del CT puede producirse durante su inserción (aspecto sobre el que no podemos actuar en la Unidad de Diálisis pero sí desde la Unidad de radiología) o posteriormente durante el uso habitual. Las puertas de entrada son el orificio de entrada en la piel y las conexiones arterial o venosa.

Pero la clave está en el manejo del punto de conexión, ya que es la principal vía de entrada de patógenos. Su contaminación provoca la colonización de la superficie interna del catéter y la formación de biofilm. Dicha contaminación puede producirse porque: 1) no se limpie correctamente la conexión y la línea de diálisis antes de conectar, 2) la conexión toque superficies no-estériles o quede expuesta al aire durante un tiempo prolongado, ó 3 ) se respire encima de ella sin mascarilla (personal de enfermería o paciente).

Según las guías de la SEN, en los pacientes en HD se aconseja el empleo de tapones de un sólo uso, con rosca de seguridad (Luer-Lock) ${ }^{4}$.

En el mercado existen además dispositivos con sistema mecánica y microbiológicamente cerrados para los catéteres venosos centrales (bioconectores tipo luer-lock). Estos dispositivos eliminan la manipulación de la conexión del catéter al acoplar o retirar líneas sanguíneas, siendo tapones que permanecen puestos durante la sesión de HD.

La ventaja que ofrecen es la menor manipulación de la luz del catéter al cambiarse sólo una vez en semana en lugar de hacerlo en cada sesión de HD. Tampoco precisan ser sustituidos cuando hay que realizar lavados de la luz del catéter, por lo que disminuye la exposición ambiental de la misma. 
En nuestra unidad partíamos de una tasa de bacteriemia muy baja, con una incidencia de 0,24/1000 días-catéter ${ }^{7}$.

En los últimos congresos de la Sociedad Española de Enfermería Nefrológica (SEDEN) se han presentado trabajos sobre la utilización de estos bioconectores. Uno de estos estudios realiza una comparación entre distintas marcas de bioconectores ${ }^{5}$ y un segundo estudio presenta resultados con uno de ellos ${ }^{6}$.

Los objetivos de nuestro estudio son:

- Analizar si hay variaciones en las presiones y flujos sanguíneos con la utilización de bioconectores en comparación con el uso habitual del catéter.

- Conocer si hay variaciones en los parámetros de eficacia dialítica medida por Kt y Kt/V.

- Conocer si hay variación en la tasa de bacteriemia relacionada con el catéter ( $B R C$ ) con el uso de bioconectores.

\section{Material y métodos}

El estudio se realizó sobre una muestra de 14 pacientes de la unidad de diálisis portadores de CT permanentes; 8 mujeres y 6 hombres. Edad media de 71,2 años (rango de 49 a 88). El tiempo medio de permanencia desde su inserción era de 13,92 meses (rango 1-51 meses).

Del total, 13 eran CT yugulares tipo permacath, 2 izquierdos y 11 derechos, y una paciente era portadora de un CT doble tipo tesio en yugular derecha.

Se excluyeron aquellos pacientes portadores de catéter permanente para hemodiálisis que utilizaron otro acceso vascular durante el tiempo de estudio.

Se trata de un estudio experimental de diseño cruzado, en el que se analizan dos periodos con 14 CT yugulares permanentes para hemodiálisis.

Periodo 1: seis sesiones consecutivas de diálisis (dos semanas) siguiendo el protocolo de conexión convencional.
Periodo 2: seis sesiones consecutivas de diálisis (dos semanas) siguiendo un protocolo de conexión con bioconectores TEGO.

Para ello se elaboró un protocolo específico para la conexión y desconexión con el uso de los bioconectores en el que se especifica cómo se realiza la manipulación y cuándo debían cambiarse (Anexo 1).

\section{ANEXO I PROTOCOLO CONEXIÓN CON EL CONECTOR TEGO}

Día uno:

1. Después de la desinfección de cada luz del catéter con una gasa impregnada con clorhexidina aplicar un bioconector de Tego a cada luz.

2. Limpiar el Tego.

3. Realizar el lavado de las vías según protocolo.

4. Limpiar el Tego.

5. Conectar las vías y realizar el tratamiento.

6. Al finalizar el tratamiento, retirar las vías.

7. Limpiar el Tego.

8. Lavar las vías del catéter y heparinizar según protocolo.

9. Cubrir el catéter con apósito.

Día tres:

1. Descubrir el catéter.

2. Limpiar el Tego.

3. Realizar el lavado de las vías según protocolo.

4. Conectar las vías y realizar el tratamiento.

5. Retirar las vías.

6. Limpiar el Tego.

7. Lavar las vías y heparinizar según protocolo.

Día cinco:

1. Descubrir el catéter.

2. Limpiar el Tego.

3. Realizar el lavado de las vías según protocolo.

4. Conectar las vías y realizar el tratamiento.

5. Retirar las vías. 


\begin{abstract}
6. Limpiar el Tego.
7. Lavar las vías y heparinizar según protocolo.

(Limpiar el Tego según protocolo: utilizar clorhexidina al $2 \%$ y realizar un movimiento circular fuerte durante tres segundos).
\end{abstract}

Precauciones: No se requiere tapar el Tego EI dispositivo está cerrado.

No utilizar agujas para conseguir el acceso.

La eficacia funcional y microbiológica de Tego ha sido valorada durante un período de hasta siete días. Cambiaremos el dispositivo Tego los lunes para pacientes L-X-V y los martes para pacientes M-J-S. Para pacientes con más de tres sesiones por semana, se cambiará el primer día de la semana de sesión. Se anotará en la gráfica (en el apartado acceso vascular) el cambio de dispositivo.

Durante estos períodos que transcurrieron entre el mes de enero y febrero del 2010 se recogieron en una hoja Excel diseñada a tal efecto, los siguientes datos: flujo de sangre, presiones arterial y venosa, eficacia dialítica medida por $\mathrm{Kt}$ y Kt/v e incidencias que se presentaron durante la diálisis relacionadas con el uso del CT (inversión de ramas, necesidad de lavados de las ramas del CT, uso de fibrinolíticos, etc...).

Así mismo, se recogieron todos los datos relacionados con la incidencia de bacteriemia durante todo el periodo de estudio.

Durante todas las fases del estudio se emplearon estrictamente las medidas de precaución universal para el manejo de CT para HD y al final de cada sesión los CT se sellaron según protocolo de nuestra unidad con 2,5 cc de heparina al $1 \%$.

Las diferencias de las variables cuantitativas en cada periodo se evaluaron usando el test $t$ de Student apareado. Todos los valores se expresan como media y desviación estándar. El valor de cada variable para cada CT es la mediana de las seis sesiones de diálisis en cada periodo. Se ha utilizado el programa SPSS 15.0 para el procesamiento de datos.

\begin{tabular}{|l|l|l|l|}
\hline & GRUPO 1 & GRUPO 2 & p \\
\hline Flujo & $331,57 \pm 42,8$ & $323,64 \pm 38,4,8$ & 0,053 \\
\hline PV & $149,37 \pm 30,4$ & $151,85 \pm 30,6$ & 0,3 \\
\hline PArt & $-185,91 \pm 27,59$ & $-190,091 \pm 21,85$ & 0,278 \\
\hline KT & $46,57 \pm 6,15$ & $45,94 \pm 5,47$ & 0,55 \\
\hline KT/V & $1,400 \pm 0,27$ & $1,3929 \pm 0,33$ & 0,83 \\
\hline
\end{tabular}

Tabla 1: Medias, Desviación Estándar y Nivel de Significación

\section{Resultados}

Como se puede ver en el gráfico 1 , no se objetivaron diferencias significativas entre las medias de los flujos sanguíneos en ambos periodos, aunque el nivel de significación está en el límite. Con respecto a las medias de las presiones arteriales y venosas tampoco se obtuvieron diferencias significativas (gráficos 2 y 3 ). En el gráfico 4 se puede ver el nivel de significación de la diferencias ente las medias de los KT obtenidos en las sesiones, en el que tampoco se objetivaron diferencias.

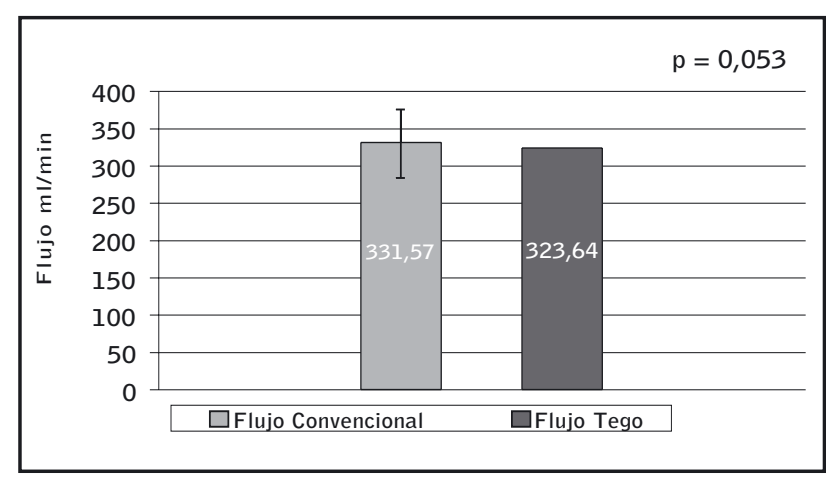

Gráfico 1: Comparación de Flujo

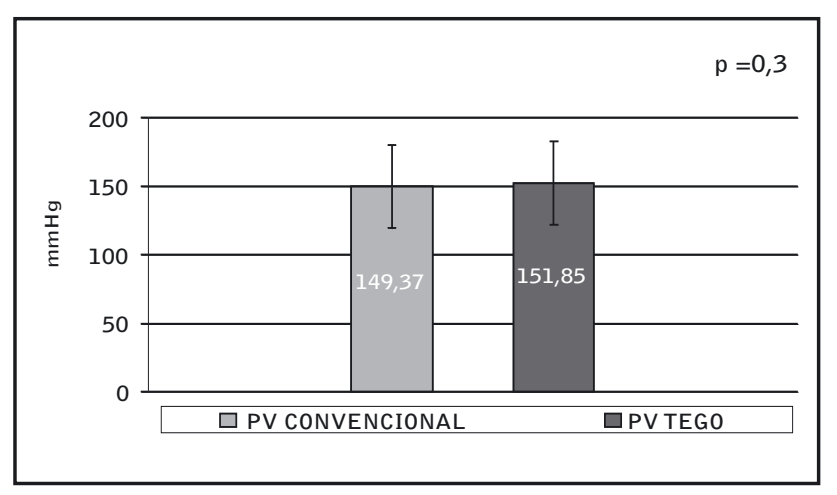

Gráfico 2: Comparación de Presión Venosa 


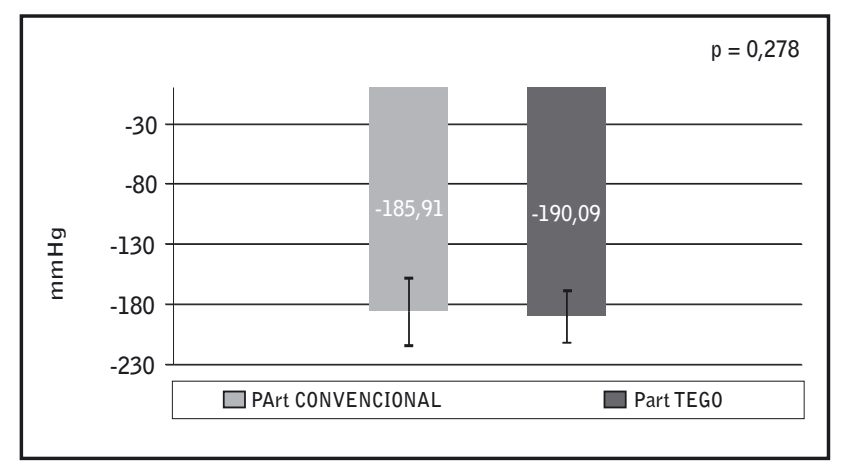

Gráfico 3: Comparación de Presión Arterial

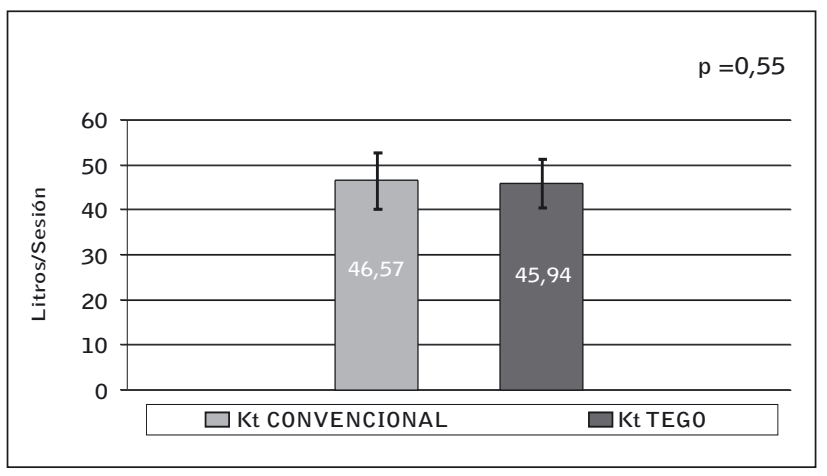

Gráfico 4: Comparación de Kt

Con respecto a las diferentes incidencias relacionadas con el CT que se presentaron durante el estudio señalar que durante el primer período (protocolo convencional) se presentaron 31 sesiones que precisaron invertir las ramas, frente a 26 sesiones durante el período TEG0 (los pacientes que precisaron invertir ramas coincidían en ambos períodos diferenciándose en el número que precisó cada uno).

Así mismo, señalar que durante el primer período se selló con fibrinolítico en 3 ocasiones frente a 2 ocasiones en el segundo período (en diferentes pacientes).

Durante el tiempo del estudio no se presentó ningún caso de BRC en ninguno de los pacientes.

\section{Discusión y conclusiones}

No se obtuvieron diferencias significativas en el flujo de sangre, presiones arterial y venosa, $\mathrm{Kt}$ y $\mathrm{Kt} / \mathrm{V}$ con el uso de los dispositivos TEGO frente al uso convencional. Podemos concluir en nuestro estudio que el uso de tapones TEGO no afecta a los parámetros dialíticos. El número y el tipo de incidencias que recogimos en ambos períodos son bastante similares e incluso algo mejores en el periodo con tapones TEGO.

Después del proceso de aprendizaje del protocolo de conexión y desconexión con el dispositivo, el $85 \%$ del equipo de enfermería que lo utilizó ( 16 enfermeros/as) refirió un alto grado de satisfacción en la utilización de los mismos, destacando como ventaja la disminución de los tiempos de conexión y desconexión.

La ventaja principal de estos sistemas es el sistema mecánica y microbiológicamente cerrado que no permite que las luces de las ramas del catéter queden en contacto con el aire.

No obstante, desde la práctica clínica habitual en nuestra unidad, debemos considerar el manejo de cualquier tipo de dispositivo como secundario en la prevención de bacteriemias relacionadas con los CT para HD. Como muestra un trabajo realizado en nuestra unidad ${ }^{7}$ es el manejo correcto del CT, haciendo especial énfasis en la asepsia durante su manipulación, la principal y mejor herramienta para evitar las bacteriemias relacionadas con los CT para HD.

Así, se ha demostrado que son las medidas universales de asepsia realizadas por un personal entrenado y bien preparado las que logran una tasa de eventos muy baja: 0,24/1000 días-catéter.

Recibido: Diciembre 2010

Revisado: Enero 2011

Modificado: Enero 2011

Aceptado: Febrero 2011

\section{Bibliografía}

1. Tordoir J, Canaud B, Haage P, Konner K, Basci A, Fouque D, et al. EBPG on vascular access. Nephrol Dial Transplant 22:88-117, 2007.

2. E. Gruss, J.Portolés, P.Caro, J.L. Merino, P. LópezSánchez, A.Tato, y cols Los modelos de atención al 
acceso vascular condicionan resultados heterogéneos en los centros de una misma comunidad. . Nefrología 30(3):310-6, 2010.

3. Registro español de Enfermos Renales. Informe 2006 de diálisis y trasplante renal en España. Nefrología 29:525-533, 2009.

4. Rodríguez Hernández JA, Gutiérrez Julián JM. Catéteres venosos centrales. Guías de acceso vascular en hemodiálisis. SEN 6:116-166, 2004.

5. Cobo Sánchez JL, Incera Setién ME, Vicente Jiménez MY y cols. Estudio comparativo de flujos y re- sistencias de catéteres para hemodiálisis usando bioconectores luer-lock. Rev Soc Esp Enferm Nefrol.13 (1): 23-28, 2010.

6. Bort Castelló J, Salvador Lengua C, Mallol Domínguez $\mathrm{A}$ y cols. Evaluación de un nuevo dispositivo de conexión. Comunicaciones presentadas al XXXIV Congreso Nacional SEDEN 2009.

7. Albalate $M$, Pérez García $R$, De Sequera $P, A l c a ́ z a r$ $R$, Puerta M, Ortega M, Mossé A, Crespo E. ¿Hemos olvidado lo más importante para prevenir las bacteriemias en pacientes portadores de catéteres para hemodiálisis?. Nefrología 30(5):573-7, 2010. 